\title{
AMEBAS TESTÁCEAS OCORRENTES NA REGIÃO DE PORTO ALEGRE, RIO GRANDE DO SUL. II. NOVOS REGISTROS PARA A REGIÃO
}

\section{Vladimir Stolzenberg Torres $^{1}$}

\begin{abstract}
Testate amoebae found in the Region of Porto Alegre, Rio Grande DO SUL. II. NEw RECORD TO THE REgION. A second study of the testate amoebae of the region of Porto Alegre, Rio Grande do Sul revealed the presence of Arcella Ehrenberg, 1830, Difflugia Leclerc, 1815, Lesquereusia Schulumberger, 1845, Centropyxis Stein, 1857, Bulinularia Penard, 1911 and Euglypha Dujardin, 1841 genera. Our finds demand emendations of the diagnostic characteristics of several species. KEY WORDS. Protista, Rhizopoda, systematics, testacean-amoebae
\end{abstract}

A presente contribuição faz parte de um amplo estudo que vem sendo desenvolvido nos últimos anos e visa complementar o trabalho publicado por TORRES \& JEBRAM (1994) sobre a procta de amebas testáceas em diferentes regiões do município de Porto Alegre, Rio Grande do Sul. Doze novos registros de ocorrência são feitos para espécies pertencentes aos gêneros Arcella Ehrenberg, 1830, Difflugia Leclerc, 1815, Lesquereusia Schulumberger, 1845, Centropyxis Stein, 1857, Bulinularia Penard, 1911 e Euglypha Dujardin, 1841.

\section{MATERIAL E MÉTODOS}

Os procedimentos para isolamento e cultivo adotados para Testacea foram os propostos por JEBRAM (1993) e TORRES \& JeBram $(1993,1994)$.

A taxonomia foi estabelecida utilizando-se animais vivos e com base em Boltovskoy (1957), VucETiCH (1973), GreEN (1975), OGden \& HEdLEy (1980); OGden (1984), MARgulis et al. (1990), TORRES \& JEBram $(1993,1994)$ e TORRES (1995, 1996).

As dimensões limites, nas características diagnósticas de cada espécie, foram baseadas em Vucetich (1973), GREEN (1975) e OGDEN \& HEDLEY (1980) acrescidas ainda de TORRES (1995).

Os locais e ambientes de coletas foram os mesmos apresentados por TORRES \& JEBRAM (1994).

1) Universidade de Caxias doSul. Rua Coronel João Pacheco de Freitas 490, 91251-060 Porto Alegre, Rio Grande do Sul, Brasil.

E-mail: pgtorres@cpovo.net 


\section{RESULTADOS}

Filo: Rhizopoda Siebold, 1845

Classe: Testacealobosea De Saedeleer, 1934

Ordem: Eulobosa De Saedeleer, 1934

Arcellidae Ehrenberg, 1830

Arcella conica (Playfair) Deflandre, 1928 (Fig. 1)

Testa formada por uma pirâmide truncada apresentando quatro faces, sobremontada por uma pirâmide mais curta, esta com quatro ou cinco faces. Vista apicalmente o contorno revela-se anguloso, visualisando-se um polígono mais ou menos regular de quatro a seis lados côncavos com ângulos arredondados e um segundo polígono com quatro a cinco faces côncavas. Piloma circular e proporcionalmente pequeno, em alguns indivíduos visualisa-se um pequeno tubo pilomar. Apresenta alréolas estremamente finas. Coloração normalmente alaranjada.

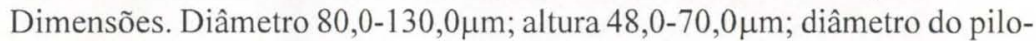
ma 20,0-45,0 $\mu$ m; altura do tubo pilomar (quando presente) $12,0-14,0 \mu$ m; relação altura/diâmetro 0,54-0,60.

Habitat. Dulceaqüícola.

\section{Arcella megastoma Penard, 1902 (Fig. 2)}

A testa apresenta o contorno circular a um pouco elíptico. O piloma é proporcionalmente muito grande, circundado por numerosos poros. A face dorsal, vista lateralmente, tem forma de um segmento de círculo. A face oral apresenta-se um pouco invaginada e sem tubo pilomar. A testa apresenta auréolas pouco visíveis e sua coloração nas águas metropolitanas tem variado do laranja até tons avermelhados.

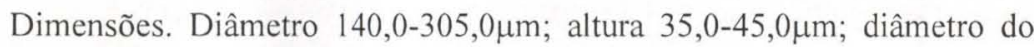
piloma 70,0-140,0 $\mu \mathrm{m}$; relação altura/diâmetro 0,15-0,25.

Habitat. Dulceaqüícola e muscícola.

\section{Difflugidae Wallich, 1864}

Difflugia mitriformis Wallich, 1864 (Fig. 3)

Testa discretamente ovalada, com a extremidade superior arredondada, as vezes apresentando um espinho (raramente dois a quatro). Extremidade oral truncada. Secção transversal circular. Parede quitinóide recoberta de partículas inorgânicas aglutinadas em quantidades variáveis; encontram-se na natureza exemplares com pouca quantidade de material aglutinado. Piloma esférico, muito raramente apresentando uma discreta elipsidade.

Dimensões. Diâmetro 23,0-24,0 $\mu$ m; altura 170,0-340,0 $\mu \mathrm{m}$; diâmetro da abertura pilomar 23,0-24,0 $\mu$ m; relação altura/diâmetro 7,40-14,17.

Habitat. Dulceaqüícola.

\section{Difflugia gramen Penard, 1902 (Fig. 4)}

Testa ovalada com paredes delgadas, relativamente transparentes, composta pela aglutinação de partículas arenáceas, unidas entre si, por uma substância 
cimentante e hialina. O piloma apresenta três lobos regulares, profundos e bem definidos. Podem ser observadas, algumas vezes, a presença de zooclorelas simbióticas no citoplasma.

Dimensões. Diâmetro 40,0-64,0 $\mu$ m; altura 51,0-90,0 $\mu$ m; diâmetro do piloma (de um lobo a base oposta) 12,0-27,0 4 ; relação altura/diâmetro 1,28-1,41.

Habitat. Dulceaqüícola.
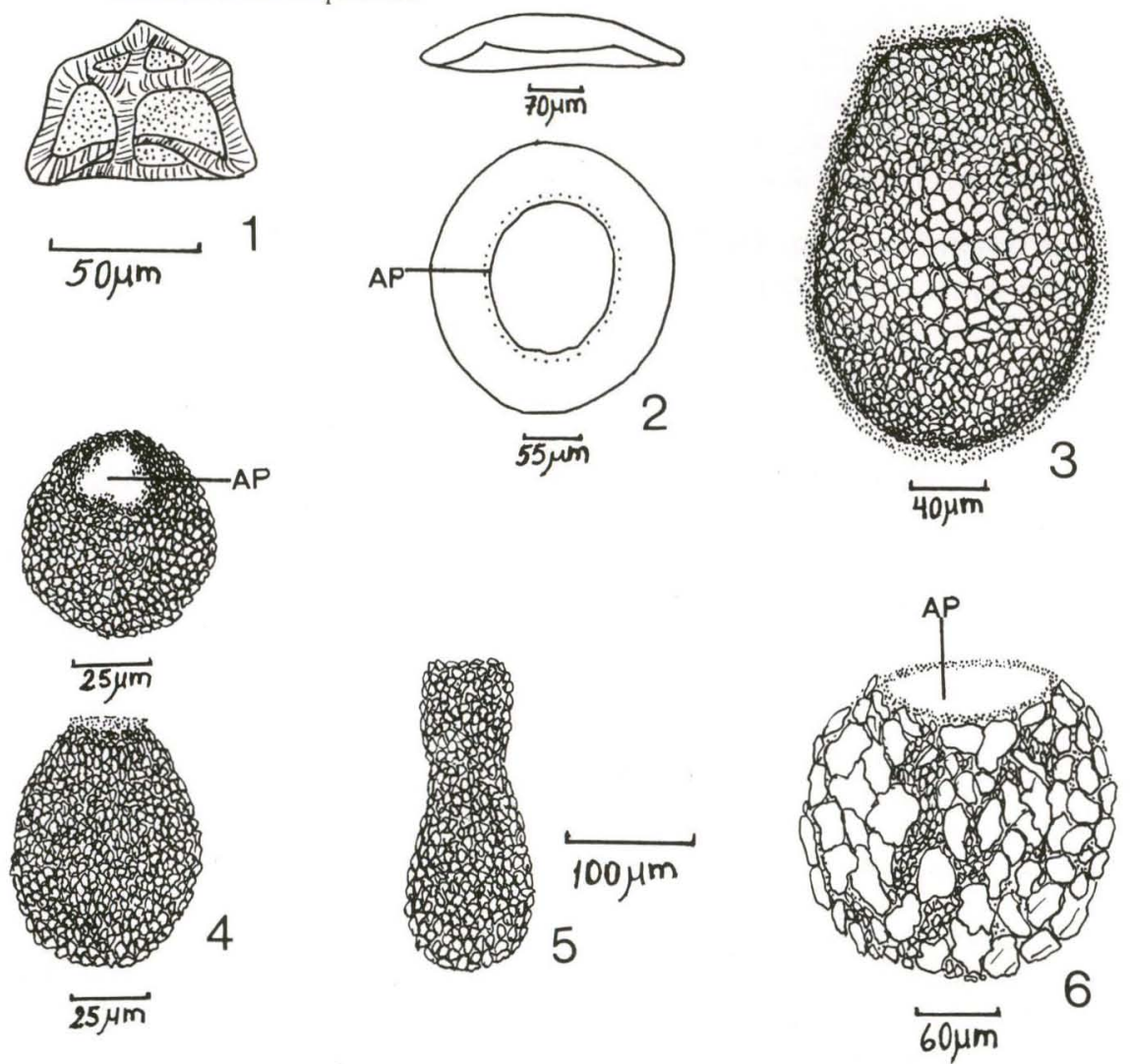

Figs 1-6. (1) Arcella conica, vista lateral; (2) Arcella megastoma, vista lateral e vista dorsal; (3) Difflugia mitriformis, vista lateral; (4) Difflugia gramen, vista oral e vista lateral; (5) Difflugia capreolata, vista lateral; (6) Difflugia aff. globularis, vista lateral. (AP) Abertura pilomar.

\section{Difflugia capreolata Penard, 1902 (Fig. 5)}

Testa piriforme apresentando seç̧ão transversal circular. Gargalo curto e largo sofrendo um estrangulamento em direção a região dorsal. Paredes da testa apresentam-se recobertas por partículas arenáceas. O piloma é grande, circular, rodeado por pequenas partículas irregulares.

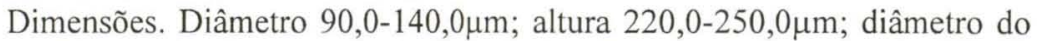
piloma 68,0-80,0 $\mu$ m; relação altura/diâmetro 1,78-2,44.

Habitat. Dulceaqüícola e muscícola. 


\section{Difflugia aff. globularis Wallich, 1864 (Fig. 6)}

Testa esferoidal ou quase esferoidal, quitinóide, aglutinada, de parede grossa, constituída por partículas minerais de diferentes classes, estas situando-se, normalmente, na faixa de $1,0-8,0 \mu \mathrm{m}$, podendo apresentar, eventualmente, partículas maiores além de frústulas de diatomáceas. Abertura irregularmente arredondada, de tamanho variável, não havendo relação definida com as dimensões da testa, algumas vezes apresenta-se relativamente pequena (até $1 / 8$ do diâmetro total da testa).

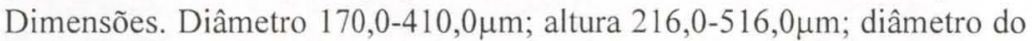
piloma 50,0-140,0 $\mu$ m; relação altura/diâmetro 1,26-1,30.

Habitat. Dulceaqüícola. Apresenta forte afinidade por rizosferas densas do tipo encontrado em Eichhornia Kunth e Salvinia Seguier, 1754.

\section{Centropyxidae Jung, 1942}

Centropyxis aculeata (Ehrenberg) Stein sec. Penard, 1902 (Figs 7e-13)

Testa apresentando contorno subcircular, comprimida na região anterior. $\mathrm{O}$ piloma é excêntrico e se encontra cercando o bordo anterior da testa. Seu contorno é geralmente irregular. A superfície da testa está normalmente recoberta, em maior ou menor grau, por partículas arenáceas, frústulas de diatomáceas e detritos, conforme a disponibilidade do material. Há casos em que a testa pode estar inteiramente recoberta por frústulas de diatomáceas. A região posterior da testa apresenta um número variável de espinhos dirigidos para cima e que apresentam, como porção terminal um pequeno bastão pontiagudo e brilhante. Conforme o aumento em que esta espécie é visualizada, tem-se a sensação de que os espinhos apresentam "unhas".

Dimensões. Diâmetro (sem espinhos) 110,0-145,0 $\mu \mathrm{m}$; comprimento dos espinhos 15,0-20,0 $\mu \mathrm{m}$; diâmetro do piloma 30,0-40,0 $\mu \mathrm{m}$.

Habitat. Parece apresentar uma forte afinidade com plantas, sendo encontrado comumente em turfeiras e, nos ambientes não túrficos, associada a rizosfera de plantas como Salvinia e Eichhornia.

\section{Centropyxis platystoma Deflandre, 1929 (Fig. 8)}

Ventralmente, o contorno da testa, apresenta-se elíptico ocorrendo uma pequena constrição entre a face ventral e o piloma. O piloma (abertura pilomar) pode ser elíptico ou circular. Lateralmente, a parte posterior de testa é elevada prolongando-se em uma espécie de viseira que recobre a abertura pilomar. A testa é quitinóide, recoberta totalmente com partículas arenáceas aglutinadas.

Dimensões. Comprimento 70,0-98,0 $\mu \mathrm{m}$; largura 42,0-68,0 $\mu \mathrm{m}$; altura igual a largura, ou pouco menor; diâmetro do piloma 24,0-36,0 $\mu \mathrm{m}$.

Habitat. Predominantemente muscícola (em Sphagnum Linnaeus, 1753) e eventualmente, dulceaqüícola em substrados sólidos como rochas e porções submersas de vegetais.

\section{Lesquereusiidae Jung, 1942}

\section{Lesquereusia modesta Rhumbler, 1896 (Fig. 9)}

Testa ovalada apresentando gargalo curto de base larga. Vista lateralmente, a separação entre o gargalo e o ventre da testa está marcado por um sulco mais ou 
menos reto. Secção de testa é elíptica. O revestimento externo da testa é composto por partículas arenáceas.

Dimensões. Comprimento 105,0-185,0 $\mu \mathrm{m}$; largura 80,0-85,0 $\mu \mathrm{m}$; diâmetro do piloma 15,0-48,0 $\mu \mathrm{m}$; espessura 45,0-55,0 $\mu \mathrm{m}$.

Habitat. Dulceaqüícola.
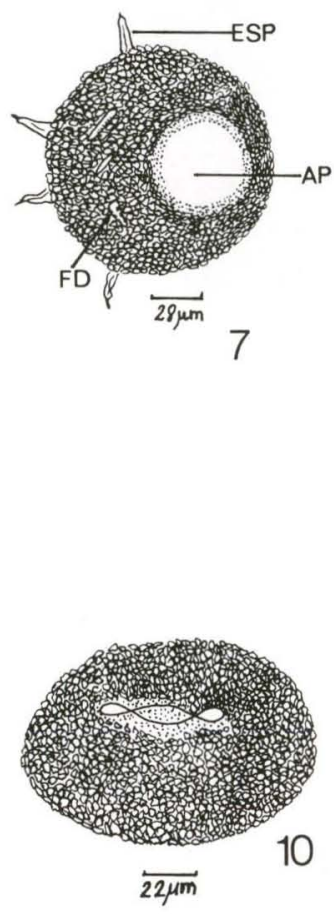
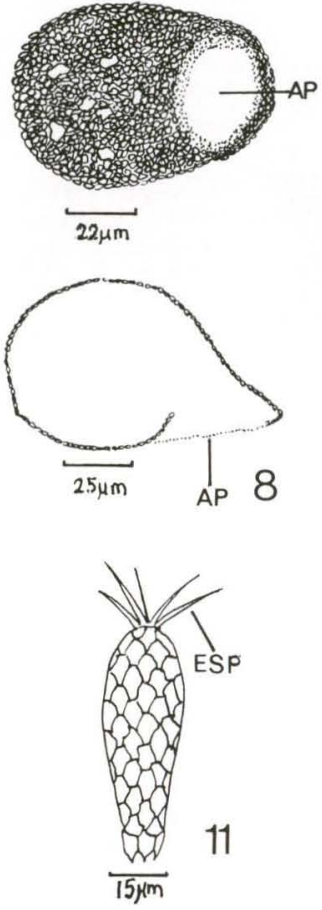
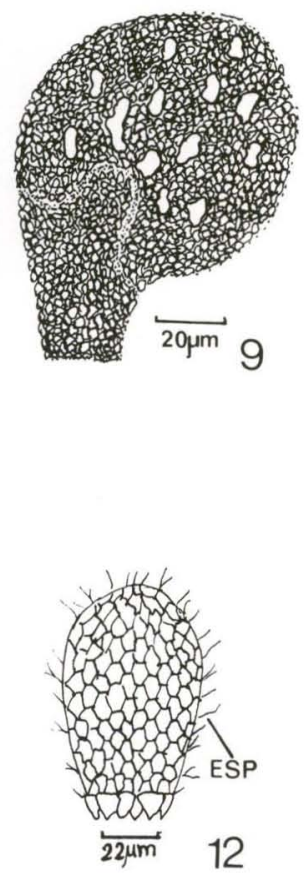

Figs 7-12. (7) Centropyxis aculeata, vista ventral esquemática; (8) Centropyxis platystoma, vista ventral e corte esquemático da vista lateral; (9) Lesquereusia modesta, vista lateral; (10) Bulinularia gracilis, vista ventral; (11) Euglypha strigosa, vista lateral; (12) Euglypha cristata, vista lateral com exposição da abertura pilomar. (AP) Abertura pilomar, (ESP) espinho.

\section{Plagiopyxidae Bonnet, 1959}

\section{Bulinularia gracilis Thomas, 1959 (Fig. 10)}

Testa em forma de elipsóide, com pouca ou nenhuma transparência, mas sempre com mais transparência do que B. indica (Penard) Deflandre, 1953. A abertura pilomar é clara e bem visível, mas os poros que a rodeiam são menos visíveis do que em $B$. indica. O revestimento é composto de placas silicosas exógenas, delgadas e remodeladas podendo estar ausentes em alguns casos.

Dimensões. Diâmetro 110,0-125,0 $\mu \mathrm{m}$; altura 60,0-82,0 $\mu \mathrm{m}$; diâmetro da abertura pilomar 11,0 x 54,0 $\mu$ m; relação altura/diâmetro 0,55-0,66.

Habitat. Dulceaqüícola. Podendo, não raramente, ser encontrada em associação com Sphagnum sp. 


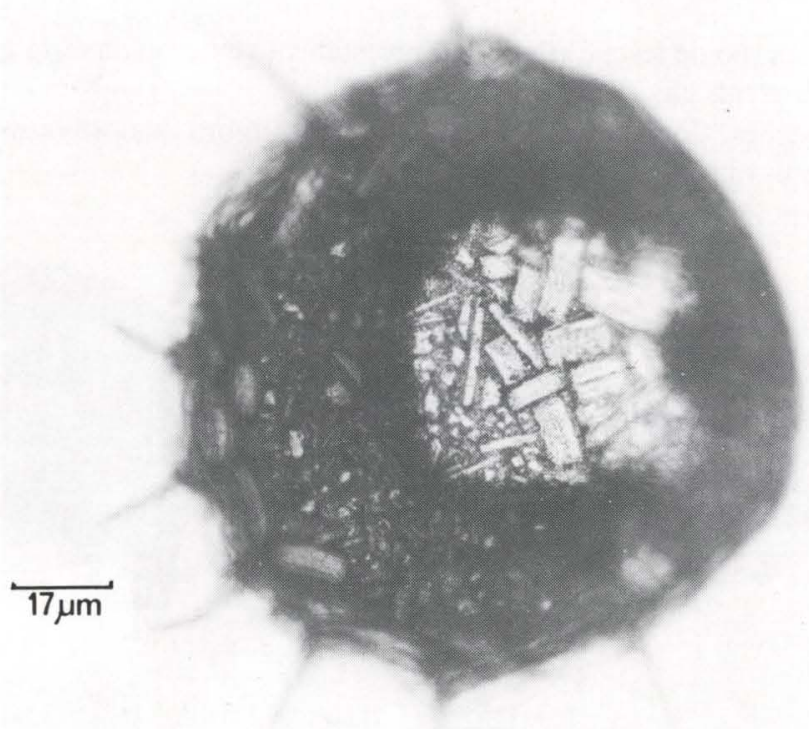

13

Fig. 13. Centropyxis aculeata, vista ventral apresentando testa constituida por frústulas de diatomáceas.

Classe: Testaceafilosea De Saedeleer, 1934

Ordem: Gromida Claparède \& Lachmann, 1878

\section{Euglyphidae Wailes, 1911}

Euglypha strigosa (Ehrenberg) Leidy, 1878 (Fig. 11)

Testa oval, com secção transversal elíptica. Adornada com vários espinhos robustos, os quais projetam-se isolados ou pareados a partir das junções das escamas. Abertura pilomar composta por 10 a 12 escamas. Escamas afiladas, em sua extremidade inferior e sendo, normalmente amplas e arredondadas na porção superior. As escamas da abertura pilomar apresentam na porção inferior um dente mediano afiado e curvado em direção ao interior da abertura pilomar, e dois a três pares laterais de dentes, os quais decrescem de tamanho do centro para as extremidades. Dois núcleos com nucléolos centrais visíveis, em indivíduos de cultivo observados em 1.200X.

Dimensões. Diâmetro 23,0/26,0 X 38,0/60,0 $\mathrm{m}$; altura 40,0-100,0 $\mu \mathrm{m}$; diâmetro da abertura pilomar 13,0 X 17,0 $\mu \mathrm{m}$; comprimento dos espinhos 2,0-3,0 $\mu \mathrm{m}$.

Habitat. Dulceaqüícola.

\section{Euglypha cristata Leidy, 1874 (Fig. 12)}

Testa oval, com secção trasversal circular. Revestida por aproximadamente 80 escamas. As escamas encontram-se arranjadas de forma regular exceto na abertura pilomar onde são mais alongadas com a extremidade projetando-se em 
dentes. Presença de espinhos longos e curvados na porção adoral da testa. A abertura pilomar apresenta-se circular e rodeada por cinco a sete escamas dentadas. Cada escama da abertura pilomar é encimada por três ou quatro escamas. Logo acima da primeira fileira de escamas que compõe a abertura pilomar observa-se uma leve constrição da testa.

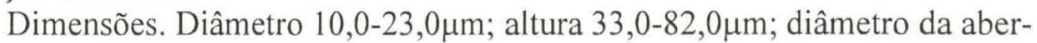
tura pilomar 6,0-16,0 $\mu \mathrm{m}$; comprimento dos espinhos 10,0-31,0 $\mu \mathrm{m}$; relação altura/diâmetro 3,30-3,57.

Habitat. Dulceaqüícola. Muito raramente foi observada em associação com a rizosfera de macrófitas aquáticas.

\section{DISCUSSÃO E CONCLUSÃO}

Nesta segunda contribuição, a espécie que apresentou maior dispersão foi Difflugia aff globularis seguida por Centropyxis aculeata.

Centropyxis aculeata desperta maior atenção devido o elevado grau de diferenciação morfológica quanto a composição da testa, como pode ser visto nas figuras 7 e 13, sugerindo, desta forma, que outros materiais disponíveis no ambiente possam ser utilizados para a confecção da mesma quando da ausência de partículas arenáceas.

São apontadas por CHARDEZ (1970), 12 variedades de Centropyxis aculeata ocorrentes em países da Europa, África e Américas Central e do Sul. Segundo este autor, as modificações da testa parecem estar associadas às variações de temperatura. Em diferentes faixas de temperatura predominam morfologias distintas da testa desta espécie.

BONNET (1975) apresenta diferentes tipos morfológicos de testas em função da ecologia e evolução mas não cita, porém, dados a respeito da composição das mesmas. Parece certo que uma espécie, salvo algum mecanismo genético intrínseco, procure utilizar o material inorgânico mais abundante e apropriado para a confecção de sua testa, o que justificaria os diferentes graus de intensidade de uso de frústulas de diatomáceas observados em C. aculeata.

Conforme CHARDEZ (1970) a ausência de certos materiais impulsiona as amebas testáceas a utilizarem abundantemente as carapaças de algas para a confecção de sua testa, o que leva a formação de uma testa muitas vezes delicada e de aspecto quitinoso.

É possível, desta forma, que outras espécies como Difflugia bacillariarum Perty, 1852, D. correntina Vucetich, 1978, D. bacillifera Penard, 1890, D. congolensis Gauthier-Lièvre \& Thomas, 1958 e Cyclopyxis bacillifera Chardez, 1965 representem sinonímias de outras espécies. Esta possibilidade, no entanto, somente poderá ser testada após estudos mais aprofundados de cada uma destas espécies, principalmente através de cultivos experimentais controlados.

\section{REFERÊNCIAS BIBLIOGRÁFICAS}

Boltovskoy, E. 1957. Contribuición al Conocimiento de las Tecamebas del Rio de La Plata. Acta.Geol.Lilloana 1: 229-313.

BonNET, L. 1975. Types Morphologiques, Écologie et Évolution de la Thèque chez les Thécamoebiens. Protistologica XI (3): 363-378. 
Chardez, D. 1970. Estudes sur Centropyxis aculeata (Ehrenberg) Stein (Protozoa, Rhizopoda, Testacea). Bull. Rech. Gembloux 5 (1/2): 76-86.

GreEN, J. 1975. Freshwater Ecology in the Mato Grosso, Central Brazil, IV: Associations of Testate Rhizopoda. Jour. Nat. Hist. 9: 545-560.

JeBRAm, D.H.A. 1993. Métodos Básicos e Novos para o Cultivo de Protistas Livres.

Comun. Mus. Ciênc. PUCRS, Porto Alegre, 50: 3-20.

Margulis, L; J.O. Corliss; M. Melkonian \& D.J. Chapman. 1990. Handbook of Protoctista. Boston, Jones and Bartlett Publishers, 914p.

OGDEN, C.G. 1984. Shell Structure of some Testate Amoebae from Britains (Protozoa, Rhizopoda). Jour. Nat. Hist. 18: 341-361.

Ogden, C.G. \& R.H. Hedley. 1980. An Atlas of Freshwater Testate Amoebae. Oxford, British Museum (Natural History), 222p.

Torres, V.S. 1995. Caracterização Morfológica e Biométrica de Três Amebas Testáceas Rizófilas. Biotemas, Florianópolis, 8 (1): 30-35.

— 1996. Vacúolos de Gás e Flutuação em Difflugia mitriformis Wallich (Protista, Rhizopoda, Testaceolobosea). Revta bras. Zool. 13 (1): 67-75.

TorREs, V.S. \& D.H.A. JEBRAM. 1993. Arcella gibbosa microsoma var.n. (Protozoa: Sarcodina, Arcellinida) Descrição e Observações Feitas em seu Cultivo. Biotemas, Florianópolis, 6 (2): 20-29.

1994. Amebas Testáceas Ocorrentes na Região de Porto Alegre, RS. Biotemas, Florianópolis, 7 (1/2): 65-78.

Vucetich, M.C. 1973. Estudio de Tecamebianos Argentinos en Especial los del Dominio Pampasico. Rev. Mus. La Plata, Sec. Z, 11 (108): 286-332.

Recebido em 07.XI.1996; aceito em 17.VI.1998. 